\title{
STABILITY OF HIGH ORDER ACCURATE DIFFERENCE METHODS FOR PARABOLIC EQUATIONS WITH BOUNDARY CONDITIONS*
}

\author{
J. M. VARAH
}

Abstract. We consider high order accurate difference schemes for second order parabolic equations in the quarter-plane $x \geqq 0, t \geqq 0$. We discuss the stability of these schemes for the mixed initial boundary value problem when particular high order accurate discrete boundary conditions are used.

1. Introduction. Consider the second order parabolic equation

$$
u_{t}=\left(a(x) u_{x}\right)_{x}
$$

for $x \geqq 0, t \geqq 0$, with initial conditions

$$
u(x, 0)=f(x)
$$

and boundary conditions

$$
\beta u_{x}(0, t)+\alpha u(0, t)=g(t) .
$$

We introduce a mesh $x_{v}=v h, v=1,2, \cdots, t_{n}=n k, n=1,2, \cdots$, with $\lambda=k / h^{2}=$ const. and consider one-step finite-difference approximations to (1) of the form

$$
\begin{aligned}
Q_{-1} v_{v}^{n+1} & =Q_{0} v_{v}^{n}, \\
v_{v}^{0} & =f(v h)
\end{aligned} \quad v \geqq 1,
$$

with

$$
Q_{-1}=\sum_{-r}^{p} c_{j}(x) E^{j}, \quad Q_{0}=\sum_{-r}^{p} a_{j}(x) E^{j}, \quad E v_{v}=v_{v+1} .
$$

We also use boundary conditions of the form

$$
v_{\mu}^{n}=\sum_{1}^{q} b_{\mu j} v_{j}^{n}+\tilde{g}_{\mu}, \quad \mu=0,-1, \cdots,-r+1 .
$$

Let $l_{2}(h)$ be the space of gridfunctions $\left\{v_{v}\right\}_{-r+1}^{\infty}$ such that

$$
\|v\|_{h}^{2}=\sum_{-r+1}^{\infty} h\left|v_{v}\right|^{2}<\infty
$$

and assume $Q_{-1}$ with (3) is uniformly invertible in $l_{2}(h)$, i.e., if

$$
\begin{aligned}
Q_{-1} w_{v} & =g_{v}, & v \geqq 1, \\
w_{\mu} & =\sum b_{\mu j} w_{j}, & \mu=0, \cdots,-r+1,
\end{aligned}
$$

then $\|w\|_{h} \leqq K_{0}\|g\|_{h}$. Then (2) and (3) can be expressed as

$$
v^{n+1}=Q v^{n}
$$

* Received by the editors September 22, 1970, and in revised form November 2, 1970.

$\dagger$ Department of Applied Mathematics, California Institute of Technology, Pasadena, California 91109. 
with $Q$ an operator on $l_{2}(h)$. Conditions for stability (in different norms) for such a scheme were given in [4] for one-step explicit schemes with constant coefficients (in the maximum norm), and in [5] for multistep schemes approximating parabolic systems with variable coefficients (in the $l_{2}$-norm). Both results involved the following conditions:

(i) the scheme applied to the pure initial value problem (Cauchy problem) is stable;

(ii) the operator $Q$ with coefficients frozen at the boundary has no eigenvalues $z$ with $|z|>1$;

(iii) some condition on the spectrum of $Q$ near $z=1$.

For the first condition, see Widlund [6]. The third condition is easily checked ; however, the second condition is often difficult to verify and we would like to discuss this here for high order accurate schemes. In particular, we shall show how to define the discrete boundary conditions (3) so that they agree with the continuous boundary conditions to any order of accuracy in $h$ and so that the scheme (4) is stable.

2. Accuracy of the difference scheme. We assume the basic difference scheme (2) has order of accuracy $s$; that is, if $u\left(x_{v}, t\right)=u_{v}(t)$ is the exact solution of (1), the local truncation error $\left\|Q_{-1} u_{v}(t+k)-Q_{0} u_{0}(t)\right\|=O\left(h^{s}\right)$. Of course, consistency demands $s>2$ as $k=O\left(h^{2}\right)$. One can make $s$ arbitrarily large by choosing a scheme with more points and matching more terms in the Taylor series expansion of the truncation error. In particular, one can obtain the order $s=2 r+1$ for an explicit scheme $\left(Q_{-1}=I\right)$ with symmetric coefficients. Moreover, these schemes are stable for $\lambda$ small enough (see Strang [3]). For constant coefficients, the $\left\{a_{j}\right\}$ satisfy

$$
\begin{aligned}
\sum_{1}^{r} j^{2 k} a_{j} & =\frac{(2 k) !}{2 k !} \lambda^{k}, \\
a_{-j} & =a_{j}, \\
a_{0} & =1-2 \sum_{1}^{r} a_{j} .
\end{aligned}
$$$$
k=1, \cdots, r
$$

To make the rate of convergence of (2) to (1) be $O\left(h^{s}\right)$, we also need the boundary conditions (3) to agree with the continuous boundary conditions to $O\left(h^{s}\right)$, and we need the scheme to be stable for the mixed problem. By order of accuracy of the boundary conditions, we mean that if we denote the continuous boundary condition in (1) by $B u=g$ and the discrete analogue (3) by $B_{h} v=g$, then $\left\|\left(B-B_{h}\right) u\right\|=O\left(h^{s}\right)$ for all solutions of the differential equation. Then an elementary extension of the Lax-Richtmyer theorem (see Kreiss and Widlund $[2$, p. 21] or Isaacson and Keller [1, p. 521]) shows the convergence rate is also $O\left(h^{s}\right)$.

We wish to examine the stability of high order accurate discrete boundary conditions, in particular, to verify condition (ii) of $\S 1$. For this the following observations are appropriate:

(i) We need only consider $g(t)=0$ in (1) and $\tilde{g}=0$ in (3) since we can add any multiple of a solution to the Cauchy problem (assumed stable). 
(ii) Only the first nonzero term in the continuous boundary condition plays a role in the stability analysis; the contribution of the remaining part (i.e., $\alpha u(0, t))$ to the difference scheme is only $O(h)$ and the stability conditions involve only $Q(0)$, the principal part. Thus we need only consider boundary conditions of the form

$$
\begin{aligned}
& u(0, t)=0 \\
& u_{x}(0, t)=0
\end{aligned}
$$

Of course the actual discrete scheme (3) used must approximate $\beta u_{x}+\alpha u=0$ to $O\left(h^{s}\right)$, but these can be taken as the same as that used for case (b) to first order in $h$ and thus the stability of the scheme depends only on the stability of the scheme for case (b).

If we use the general form (3) and define our accurate discrete boundary condition by equating Taylor series terms, the coefficients become quite complicated and it is difficult to verify the conditions of Lemma 1 below. And for variable $a(x)$, simplification of these coefficients using the continuous solution is also difficult. However, for $a(x)=$ const., there are simple discrete analogues which are accurate to any order:

(a) for $u(0, t)=0$, use

$$
\begin{aligned}
v(0, t) & =0, \\
v_{\mu}(0, t) & =-v_{-\mu}(0, t), \quad \mu=-1, \cdots,-r+1 .
\end{aligned}
$$

As the continuous solution is an odd function, these boundary conditions are accurate to any order.

(b) For $u_{x}=0$, we can use the fact that the continuous solution is now even, and take care of $v_{0}$ in one of two ways:

$$
\begin{array}{ll}
v_{0}=\sum_{1}^{r} b_{j} v_{j}, & \\
v_{\mu}=v_{-\mu}, & \mu=-1, \cdots,-r+1,
\end{array}
$$

with $b_{j}=2(-1)^{j-1}(r !)^{2} /((r+j) !(r-j) !)$. This gives $O\left(h^{2 r+1}\right)$ accuracy.

$$
v_{\mu}=v_{-\mu}, \quad \mu=-1, \cdots,-r,
$$

with mesh shifted by $h / 2$ in $x$-direction; then no condition is needed at $x=0$.

3. Stability of the mixed problem. In this section we shall show the boundary conditions (A) and (B2) give schemes which have no eigenvalues outside the unit circle. For this, we need a characterization of such eigenvalues.

LEMMA 1. Consider the scheme (2), (3) with constant coefficients and stable for the Cauchy problem. Then $z$ with $|z|>1$ is an eigenvalue of $Q$ if and only if $\operatorname{det} B(z)=0$, where

$$
B_{i j}=\tau_{j}^{-i+1}-\sum_{m=1}^{q} b_{-i+1, m} \tau_{j}^{m}, \quad 1 \leqq i, j \leqq r
$$


and where $\left\{\tau_{j}\right\}_{1}^{r}$ are the roots of

$$
z \sum_{-r}^{p} c_{j} \tau^{j}=\sum_{-r}^{p} a_{j} \tau^{j}
$$

inside the unit circle (with suitable modifications in B for multiple roots $\tau_{j}$ ).

Proof. If $z$ is an eigenvalue with vector $g \in l_{2}(h)$, then

$$
z \sum_{j=-r}^{p} c_{j} g_{v+j}=\sum_{-r}^{p} a_{j} g_{v+j}, \quad v=1,2, \cdots,
$$

and

$$
g_{\mu}=\sum_{1}^{q} b_{\mu j} g_{j}, \quad \quad \mu=0,-1, \cdots,-r+1 .
$$

Thus $g_{v}=\sum_{\left|\tau_{i}\right| \leqq 1} d_{i} \tau_{i}^{v}$, where $\left\{\tau_{i}\right\}$ are the roots of (8). Now since the Cauchy problem is stable,

$$
\left|\frac{\sum a_{j} e^{i j \theta}}{\sum c_{j} e^{i j \theta}}\right| \leqq 1<|z|
$$

and thus there are no $\tau_{i}$ with $\left|\tau_{i}\right|=1$ for any $|z|>1$. And for $|z| \rightarrow \infty$, it is easy to see there are the same number of $\tau_{i}$ with $\left|\tau_{i}\right|<1$ as there are for $\sum_{-r}^{p} c_{j} \tau^{j}=0$. But this has exactly $r$ such roots, since if there were more or less, the operator $Q_{-1}$ or $Q_{-1}^{-1}$ would have an eigenvalue at zero. Then the $r$ remaining homogeneous equations giving the discrete boundary conditions in the $r$ unknowns $\left\{d_{i}\right\}$ require $\operatorname{det} B(z)=0$ for solution. The converse follows similarly as in Lemma 1 of [4].

To ensure stability of the mixed problem, we must also satisfy the third condition mentioned in $\S 1$. For this, the following is sufficient:

(i) for $z \rightarrow 1, \tau_{i}(z) \rightarrow \tau_{i}(1)$ inside the unit circle,

(ii) for $z \rightarrow 1,\left\|B^{-1}(z)\right\|_{\infty}=O\left((z-1)^{-1 / 2}\right)$.

THEOREM 1. For the discrete boundary conditions (A) or (B2), the operator $Q$ has no eigenvalues $z$ with $|z|>1$. If (2) is also consistent with (1) and satisfies (i) above, then the scheme for the mixed problem is stable in the maximum norm and in the $l_{2}$-norm of [5].

Proof. Case (A). Here $B(z)$ has as its $j$ th column

$$
\left(1, \tau_{j}+\tau_{j}^{-1}, \tau_{j}^{2}+\tau_{j}^{-2}, \cdots, \tau_{j}^{r-1}+\tau_{j}^{1-r}\right)^{T}
$$

if all $\tau_{j}$ are distinct. Then $\operatorname{det} B(z)=\operatorname{det} C(z)$, where we arrive at $C$ by the following row operations : for $k=r, r-1, \cdots, 3$,

$$
\text { row } k \rightarrow \text { row } k+\left(\begin{array}{c}
k-1 \\
1
\end{array}\right)([k-2] \text { nd row })+\left(\begin{array}{c}
k-1 \\
2
\end{array}\right)([k-4] \text { th row })+\cdots
$$

These row operations transform the $k$ th row of $B$ into

$$
\left(\left(\tau_{1}+\tau_{1}^{-1}\right)^{k-1},\left(\tau_{2}+\tau_{2}^{-1}\right)^{k-1}, \cdots,\left(\tau_{r}+\tau_{r}^{-1}\right)^{k-1}\right) .
$$

Now if we let $x_{j}=\tau_{j}+\tau_{j}^{-1}, C(z)$ is a Vandermonde matrix in the $\left\{x_{j}\right\}$. Moreover, $x_{i} \neq x_{j}$ for $i \neq j$ since the $\left\{\tau_{j}\right\}$ are distinct, $\tau+\tau^{-1}$ is one-to-one inside the unit circle, and all $\left|\tau_{j}\right|<1$ for $|z|>1$. Thus $\operatorname{det} B(z) \neq 0$ for $|z|>1$ if the $\left\{\tau_{j}\right\}$ are distinct. 
Now consider multiple $\left\{\tau_{j}(z)\right\}$ for $|z|>1$, i.e., suppose $\tau_{1}(z)=\tau_{2}(z)=\tau$, and $\tau_{3}, \cdots, \tau_{r}$ are distinct. Then the general solution for $g_{v}$ in Lemma 1 is

$$
g_{v}=d_{1} \tau^{v}+d_{2} v \tau^{v-1}+\sum_{i=3}^{r} d_{i} \tau_{i}^{v}
$$

giving $B(z)$ as in (9) except its second column is

$$
\left(0,1-\tau^{-2}, 2 \tau-2 \tau^{-3}, \cdots,(r-1) \tau^{r-2}-(r-1) \tau^{-r}\right)^{T},
$$

so $b_{j 2}(\tau)=(d / d \tau)\left(b_{j 1}(\tau)\right)$. Now apply the same row operations described above, forming $C(z)$. Then we still have

$$
\begin{aligned}
c_{j 2}(\tau) & =\frac{d}{d \tau}\left(c_{j 1}(\tau)\right) \\
& =\frac{d}{d \tau}\left(x^{j-1}\right)=(j-1) x^{j-2}\left(1-\tau^{-2}\right),
\end{aligned}
$$

where $x=\tau+\tau^{-1}$. Now if we factor $\left(1-\tau^{-2}\right)$ from the second column, the remaining matrix is a confluent Vandermonde, with nonzero determinant since $x_{i} \neq x_{j}$. A similar analysis holds for other multiple $\tau_{j}(z)$. So we have in general $\operatorname{det} B(z) \neq 0$ for $|z|>1$.

For $z \rightarrow 1$, consistency implies that exactly one root $\tau_{1}(z) \rightarrow 1$ from inside the unit circle; in fact,

$$
\tau_{1}(z)=1-\alpha \sqrt{z-1}+O(z-1)
$$

for $\alpha \neq 0$ (see [4] or [5]). The other $\tau_{j}(z) \rightarrow \tau_{j}(1)$ which are inside the unit circle; if these are distinct, $C(z) \rightarrow C(1)$, which is a Vandermonde matrix with $\operatorname{det} C(1) \neq 0$. So $\operatorname{det} B(z)$ is actually bounded as $z \rightarrow 1$ and (ii) holds trivially. If the other $\tau_{j}(1)$ are not distinct but are inside the unit circle, $C(1)$ is again a confluent Vandermonde with $\operatorname{det} \neq 0$.

Case (B2). Here $B(z)$ has as its $j$ th column

$$
\left(\tau_{j}-\tau_{j}^{-1}, \cdots, \tau_{j}^{r}-\tau_{j}^{-r}\right)^{T}
$$

and if we factor $\left(\tau_{j}-\tau_{j}^{-1}\right)$ out of the $j$ th column, $j=1, \cdots, r$, the remaining matrix can be transformed into the Vandermonde or confluent Vandermonde $C(z)$ as before. Thus

$$
\operatorname{det} B(z)=\prod_{j=1}^{r}\left(\tau_{j}-\tau_{j}^{-1}\right) \operatorname{det} C(z)
$$

and this is nonzero for $|z|>1$ since $\left|\tau_{j}\right|<1$. For $z \rightarrow 1$, using (10) we have $\operatorname{det} B(z)=K \alpha \sqrt{z-1}+O(z-1)$, so again (ii) holds and the scheme is stable.

For the scheme (B1), we cannot prove it is stable for general $r$, although the question is only academic since (B2) can be used just as easily. The matrix $B(z)$ for the scheme (B1) has columns

$$
\left(1-\sum_{1}^{r} b_{j} \tau^{j}, \tau-\tau^{-1}, \cdots, \tau^{r-1}-\tau^{1-r}\right)^{T}
$$


and it seems to be quite difficult to check whether $\operatorname{det}(B)=0$ for $|z|>1$. The case $r=2$ is easy:

$$
\begin{aligned}
\operatorname{det} B(z)= & K \cdot \operatorname{det}\left(\begin{array}{lr}
\left(3-\tau_{1}\right)\left(1-\tau_{1}\right) & \left(3-\tau_{2}\right)\left(1-\tau_{2}\right) \\
\tau_{1}-\tau_{1}^{-1} & \tau_{2}-\tau_{2}^{-1}
\end{array}\right) \\
= & 0 \quad \text { if and only if } \tau_{1}=1 \quad \text { or } \tau_{2}=1 \quad \text { or } \tau_{1}=\tau_{2} \\
& \text { or } \tau_{2}=\frac{3-\tau_{1}}{1+\tau_{1}}
\end{aligned}
$$

and the last means $\left|\tau_{2}\right|>1$ since $\left|\tau_{1}\right|<1$, so this is impossible. We conjecture this scheme is stable for general $r$.

\section{REFERENCES}

[1] E. IsaAcSon And H. B. Keller, Analysis of Numerical Methods, John Wiley, New York, 1966.

$\lceil 2\rceil$ H. O. KREISS AND O. WIDlund, Difference approximations for initial value problems for partial differential equations, Rep. 7, Uppsala University Computer Science Department, Uppsala, Sweden, 1967.

[3] W. G. Strang, Accurate partial difference methods I: Linear Cauchy problems, Arch. Rational Mech. Anal., 12 (1963), pp. 392-402.

[4] J. M. VARAH, Maximum norm stability of difference approximations to the mixed initial boundary value problem for the heat equation, Math. Comp., 24 (1970), pp. 31-44.

[5] - Stability of difference approximations to the mixed initial boundary value problems for parabolic systems, this Journal, 8 (1971), pp. 598-615.

[6] O. B. Widlund, Stability of parabolic difference schemes in the maximum norm., Numer. Math., 8 (1966), pp. 186-202. 